\title{
Sur la présence d'un champignon du genre Coelomomyces en République de Haute-Volta
}

\author{
par F. RODHAIN \\ (Travail du Laboratoire de Parasitologie de la Faculté de Médecine de Paris \\ Dir. $\mathrm{P}^{r}$ M. LARIVIÈRE)
}

\begin{abstract}
Résumé
L'auteur rapporte un cas de parasitisme d'une larve d'Anophèles par Coelomomyces africanus, en Haute-Volta. Description du champignon parasite et discussion du rôle pathogène éventuel.
\end{abstract}

\section{Summary}

The author records a case of C. africanus parasitic in Anopheles larva in Upper Volta. Description of the parasite fungus. Its pathogenic importance is discussed.

En examinant un grand nombre de larves de Culicides provenant de plusieurs localités d'Afrique de l'Ouest à la recherche d'éventuels parasites, il nous a été donné d'observer une larve d'Anopheles parasitée par un champignon appartenant au genre Coelomomyces.

\section{Hôte ET LOCALITE :}

Il s'agit d'une larve jeune $\left(2^{\circ}\right.$ stade ou $3^{\circ}$ stade jeune) $d^{\prime}$ Anopheles.

Le jeune âge de cette larve, son assez mauvais état de conservation ainsi que la présence même du champignon parasite en rendent très difficile la détermination précise, mais il y a tout lieu de penser qu'il s'agit d'une larve d'A. squamosus Théo, 1901.

Cette larve fut récoltée au cours de captures systématiques à Tingréla (cercle de Banfora, Haute-Volta), le 12 juillet 1967. 
Le PARASITE :

Selon le Professeur J. N. Couch, de l'Université de Caroline du Nord (U.S.A.), qui a bien voulu se charger de la détermination de ce champignon, l'espèce en cause est $C$. africanus Walker.

Comme tous les Coelomomyces, ce champignon se présente sous l'aspect d'un mycélium non cloisonné, ramifié, flottant dans la cavité coelomique de la larve en adhérant partiellement à la paroi du tube digestif. Les sporanges, qui mesurent habituellement 25 à $35 \mu$ de long, emplissent rapidement tout le corps de la larve, y compris les papilles anales et la capsule céphalique.

Dans le cas que nous rapportons ici, les seuls éléments visibles sont des sporanges. Très nombreux, ils remplissent totalement la larve, « comme un sac de noix » (Fig. 1). Quelques éléments sont visibles dans la capsule céphalique (Fig. 2). La taille moyenne de ces sporanges (mesurée sur 100 éléments consécutifs) est de 25,75 $\mu \times 15,35 \mu$ (Fig. 3).

Cette espèce, dont les sporanges présentent une paroi ornée extérieurement de cannelures anastomosées, appartient donc au second des groupes distingués par Rioux et Pech en 1960.

Ne parasitant, semble-t-il, que les Culicides du genre Anopheles, il s'agit d'un Coelomomyces relativement fréquent déjà récolté plusieurs fois en Afrique de l'Ouest. Ce fut en effet Walker qui, le premier, en 1938, faisait mention de ce champignon chez les larves, des nymphes et un imago mâle d'A. gambiae Giles, 1902, ainsi que des larves d' $A$. funestus Giles, 1900, en Sierra Leone. Dans leur excellente revue publiée en 1953, Couch et Umphlett citent plusieurs auteurs ayant observé cette espèce chez $A$. funestus au Libéria (Gilioli et al., 1956 ; Darwish et al., 1960). D'autre part, ce parasite est également connu d'Afrique Orientale, chez $A$. gambiae et $A$. funestus au Kenya (Haddow, 1942), A. squamosus (Théo, 1901), à Madagascar (Grjebine et al., 1953), et peut-être en Angola. Enfin, en Ouganda, Gibbins (1932) aurait trouvé un champignon rapporté de façon incertaine à $C$. africanus chez $A$. gambiae.

Nous sommes par conséquent en présence d'une espèce éthiopienne du genre Coelomomyces, espèce qui semble largement répandu en Afrique, au sud du Sahara.

\section{RôLe PATHOGÈNE :}

Que doit-on penser du rôle pathogène de $C$. africanus?

D'une façon générale, le rôle pathogène des différentes espèces de Coelomomyces apparaît extrêmement variable selon les observations. Dans la plupart des cas, le champignon est observé chez un nombre très restreint de larves et le rôle pathogène, s'il est important chez l'individu infecté, est quasi nul dans la population culicidienne. C'est le cas de notre observation ou, sur plusieurs centaines de larves examinées provenant de la même localité, une seule fut trouvée porteuse d'une infection apparente.

Quelques publications toutefois font état d'une fréquence momentanément beaucoup plus élevée du champignon dans la population locale de moustiques où tous les stades évolutifs sont touchés et où, par conséquent, le rôle pathogène est très impor- 


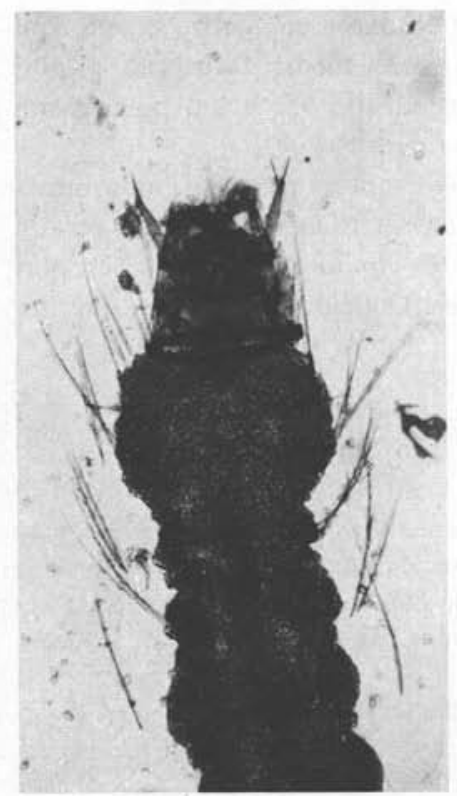

Fig. 1. - Aspect général de la larve remplie de sporanges

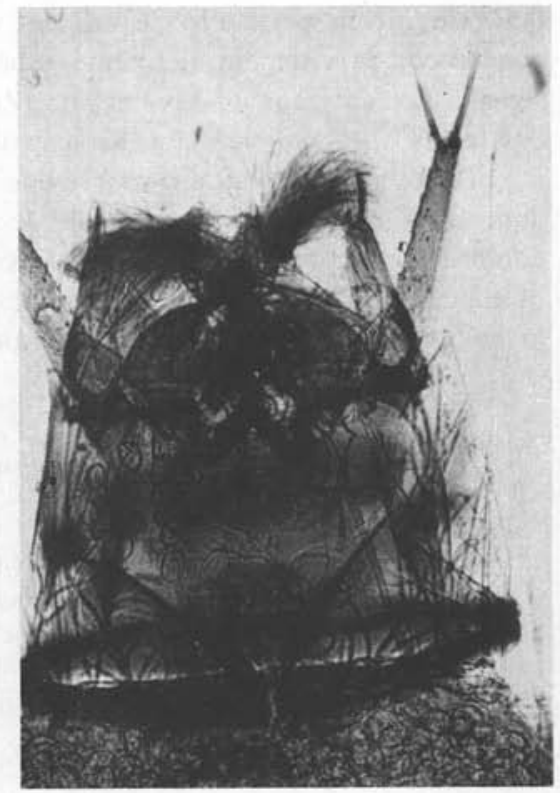

Fig. 2. - Capsule céphalique. Noter la présence de quelques sporanges à l'intérieur de la capsule

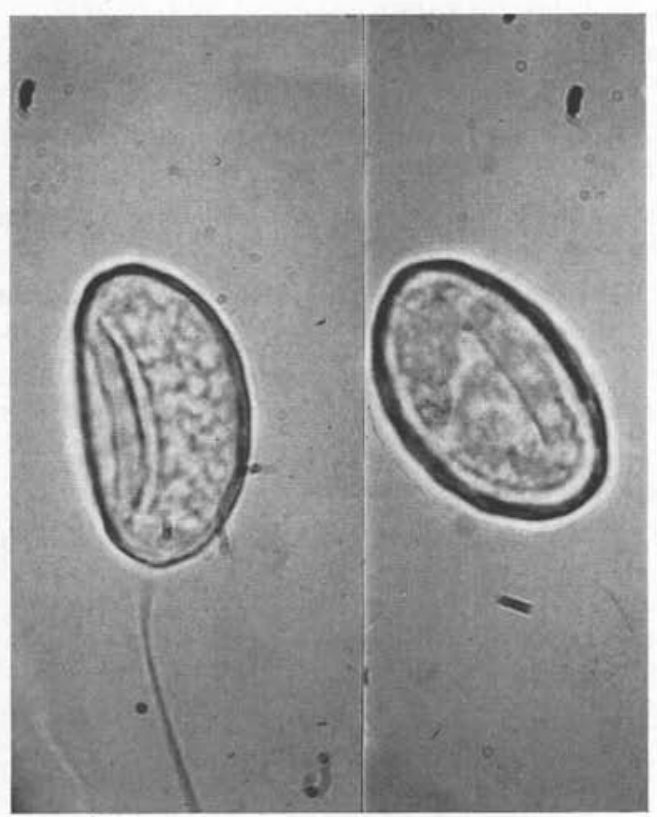

FIg. 3. - Deux sporanges isolés, vus au fort grossissement 
tant. Ce fut en particulier le cas des observations de Walker, en Sierra Leone, qui concernent précisément la même espèce $C$. africanus. De même, Muspratt (1946) signale aux environs de Livingstone (Zambie) une mortalité de $95 \%$ ou plus, parmi des larves d' $A$. gambiae, due au parasitisme par $C$. indicus.

De telles observations sont évidemment fort intéressantes chez des moustiques dont on connaît par ailleurs le rôle de premier plan dans la transmission d'un certain nombre d'affections humaines. C'est ce qui place les Coelomomyces parmi les plus prometteurs des agents de lutte biologique contre les Culicides, une fois que les mycologistes en auront réussi la culture.

\section{REMERCIEMENTS}

Nous tenons à adresser nos vifs remerciements à :

M. J. Hamon, Directeur du Laboratoire d'Entomologie du Centre Muraz, Bobo-Dioulasso (Haute-Volta), qui nous a permis d'examiner systématiquement les larves récoltées par le personnel de son laboratoire.

M. le $\mathrm{P}^{r}$ J.-N. Couch qui a bien voulu effectuer la détermination précise du champignon.

\section{Bibliographie}

Couch (J. N.) et Umphlett (C. J.), 1963. - Coelomomyces infections. In : Steinhaus E. A.: Insect Pathology, an advanced treatise, New-York \& London. Academic Presse, 2, pp. $149-188$.

GibBins (E. G.), 1932. - Natural malaria infection of house frequenting Anopheles mosquitoes in Uganda. Ann. Trop. Med. Parasit., 26, pp. 239-266.

Haddow (A. J.), 1942. - The mosquito fauna and climate of native huts at Kisumu, Kenya. Bull. Ent. Res., 33, pp. 91-142.

Muspratt (J.), 1946. - On Coelomomyces fungi causing high mortality of Anopheles gambiae larvae in Rhodesia. Ann. Trop. Med. Parasit., 40, pp. 10-17.

Rioux (J.-A.) et Рech (J.), 1960. - Coelomomyces grassei n. sp., parasite d'Anopheles gambiae Giles (note préliminaire). Acta Tropica (Basel), 17, pp. 179-182.

Rodhain (F.), 1968. - Eléments d'une révision des champignons du genre Coelomomyces, parasites des moustiques. Thèse Médecine, Paris.

WALKer (A. J.), 1938. - Fungal infections of mosquitoes, especially of Anopheles costalis. Ann. Trop. Med. Parasit., 32, pp. 231-244. 